\title{
The evolution of triaxial molecular cores
}

\author{
Simon P. Goodwin, Anthony P. Whitworth \& Derek Ward-Thompson \\ Dept. Physics \& Astronomy, Cardiff University, 5 The Parade, Cardiff, \\ CF24 3YB, UK
}

\begin{abstract}
.
We are using SPH to model the evolution of triaxial molecular cores to investigate the fragmentation of these cores and the possible production of multiple systems. Molecular cores appear to be triaxial in shape and this may have a significant effect on their evolution as they collapse to form filament-like structures which can then fragment into multiple 'protostars'.
\end{abstract}

\section{Introduction}

Stars form from dense molecular cores in giant molecular clouds, but many of the details of their formation are unknown. In particular it is not clear how multiple systems are formed.

The dense molecular cores from which stars form are thought to be triaxial in shape (eg. Jones, Basu \& Dubinski 2001; Goodwin, Ward-Thompson \& Whitworth 2001). The triaxiality of cores is also not a constant, cores having a range of axial ratios. It is reasonable to think that the triaxiality of a core might have an effect on the evolution of the core as it collapses.

We are in the process of modeling the collapse of triaxial cores and examining the fragmentation and instabilities in the cores.

\section{Simulations}

The structure of the cores is based on observed cores (mainly the typical core L1544). Central densities are around $3 \times 10^{-18} \mathrm{~g} \mathrm{~cm}^{-3}$, core radii of around 5000 AU, total masses of a few $M_{\odot}$ and based around a Plummer-like sphere with an outer fall off as $r^{-4}$ out to a limit of about $40000 \mathrm{AU}$. An initially spherical Plummer-like core is stretched to the desired triaxiality. Cores appear to have triaxialities in the range $1: 0.5-0.9: 0.3-0.6$.

Additional complications such as a bulk rotation or a 'lumpy' density field within the core may be added. This vastly increases the parameter space to be explored but help the multiple fragmentation of cores. 


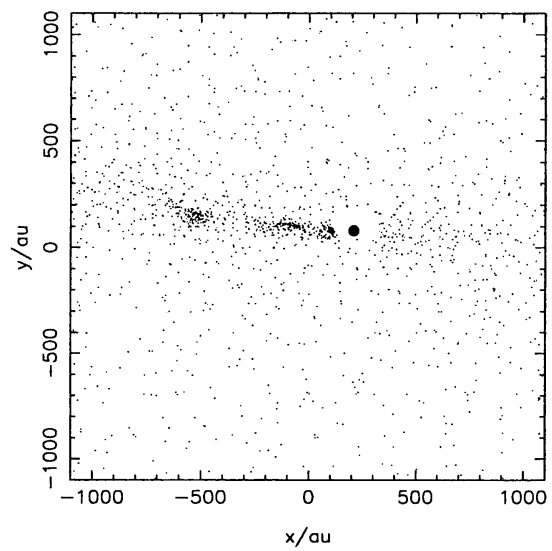

Figure 1. A $1: 0.8: 0.3$ triaxial cloud 50000 years after collapse starts.

\section{First results}

As a triaxial core collapses the collapse occurs at different rates along different axes. The short axis collapses first, creating a filament-like structure at the heart of the core. This filament can then fragment into several Jeans unstable regions which collapse.

Figure 1 shows an initially $1: 0.8: 0.3$ triaxiality cloud 50000 years after the start of collapse. The filament-like structure across the middle of the cloud is obvious and one region has collapsed and been replaced with a sink particle (the large blob) and to the left of this another region is starting to become Jeans unstable.

Once formed these regions (which we associate with protostars) accrete mass rapidly. Initial infall rates of a little less than $10^{-4} M_{\odot} \mathrm{yr}^{-1}$ are not uncommon, causing protostars to grow to $0.5 M_{\odot}$ in only 6000 years. Such a fast initial accretion rate is in good agreement with the accretion rate suggested for protostars by Whitworth \& Ward-Thompson (2001).

This work is still at a very early stage, especially as we are currently writing and testing a new SPH code, DRAGON, designed for star formation simulations in particular. We hope to release DRAGON to the community after test in complete, with any luck in early 2002 .

\section{References}

\section{References}

Goodwin, S. P., Ward-Thompson, D. \& Whitworth, A. P. 2001, MNRAS, submitted

Jones, C. E., Basu, S. \& Dubinski, J. 2001, ApJ, 551, 387

Whitworth, A.P. \& Ward-Thompson, D. 2001, ApJ, 547, 317 